\title{
Deformations of a 2D Elastica under a random distribution of normal loads
}

\author{
Miguel Trejo ${ }^{1, *}$, Arnaud Lazarus $^{2}$, Damien Vandembroucq ${ }^{3}$, and Evelyne $\mathrm{Kolb}^{3, * *}$ \\ ${ }^{1}$ Instituto de Fisica de Buenos Aires (IFIBA-CONICET), Departamento de Fisica, Facultad de Ciencias Exactas y Naturales, Universi- \\ dad de Buenos Aires, Ciudad Universitaria, Buenos Aires, Argentina \\ ${ }^{2}$ Sorbonne Université, UPMC Univ Paris 06, CNRS, UMR 7190, Institut Jean Le Rond d'Alembert, 75005, Paris, France \\ ${ }^{3}$ PMMH, CNRS, ESPCI Paris, Université PSL, Sorbonne Université, Université de Paris, F-75005, Paris, France
}

\begin{abstract}
We describe the deformations of a 2D elastic structure (beam, rod or filament) subjected to randomly distributed local orthogonal forces. The fiber is in quasistatic equilibrium condition when a given force distribution is acting on it. To analyze the effects of force fluctuations on the observed configurations, we study the behavior of the bending moment at the origin and the filament curvature, as a function of nominal values of the local mean forces and small, moderate and large fluctuations around them.
\end{abstract}

\section{Introduction}

The mechanical interaction of a slender and elastic object ('fiber') with an external constraining medium (solid, soft gel, granular, viscous or turbulent flowing liquids) occurs in many situations. Examples can be found in natural and engineering fields ([1] and references therein) such as the penetration of long drill pipes in sea beds for petroleum recovery, the insertion of long thin guide wires for investigation and surgery in medicine, the growth of plant roots in soils [2] or even at a smaller scale the development of cytoskeleton microtubules in living cells [3] or the DNA packaging in cell nuclei [4]. In all these situations, the interplay between the elasticty of the fiber and the external forces leads to a large variety of shapes and behaviors. The case of a fiber embedded inside a granular medium is of particular interest, then it deals with discrete and heterogeneous sets of forces along the fiber (See Fig. 1(a)). This situation has been investigated experimentally in [5] but almost no modeling takes into account the role of the noise in the bending behaviors of Elastica, namely the force fluctuations around an average value set by the approach to the jamming transition in the case of a granular medium.

In this work we describe the influence of force intensity fluctuations on the configurations adopted by a 2D elastic fiber subjected to random orthogonal forces. We numerically solve the equation describing the fiber shape for a particular distribution of forces and we analyze our results as a function of the force amplitude fluctuations and the beam curvature.

\section{Modeling the fiber deflection}

\subsection{Theoretical description}

To describe the planar deformation of the fiber we use the 2D Kirchhoff equations. Thus, we consider the fiber as an

\footnotetext{
*e-mail: miguel.trejo.1@gmail.com

**e-mail: evelyne.kolb@upmc.fr

A video is available at https://doi.org/10.48448/vw6p-6984
}

inextensible elastic filament of length $L$, parametrized by its arclength $s$ and clamped at the origin (See Fig. 1(b)). We assume that this fiber is subjected to internal contact forces described by $\vec{F}(s)$ and also external forces, whose amplitude by unit length is expressed by the density $\vec{f}_{e}(s)$. By balancing the force and moment acting on the fiber, we have $[6,7]$ :

$$
\begin{gathered}
\partial_{s} \vec{F}(s)+\overrightarrow{f_{e}}=0, \\
m^{\prime}(s)+(\hat{t}(s) \wedge \vec{F}(s)) \cdot \hat{k}=0 .
\end{gathered}
$$

In these equations $m(s)$ represents the internal moment in the fiber, $\hat{t}(s)$ the local unit vector tangent to the fiber and $\hat{k}$ the unit vector perpendicular to the deflection plane (Fig. 1(b)). The above system can be reduced by integrating Eq. 1 to find $\vec{F}(s)$ and by replacing this variable into Eq. 2. If we suppose that the fiber behaves as a linear elastic material, the moment is proportional to the rod curvature: $m(s)=E I \kappa(s)$ (Euler-Bernoulli law), where $E$ is the fiber elastic modulus, $I$ its moment of inertia and $\kappa(s)=\theta^{\prime}(s)$. In this work, we focus on the case of a distribution of orthogonal local forces, compatible with the experimental results obtained for a fiber embedded in grains [5], then: $\vec{f}_{e}(s)=f(s) \sin \theta(s) \hat{i}-f(s) \cos \theta(s) \hat{j}$, and the reduced moment equation is expressed as follows:

$$
\begin{array}{r}
E I \theta^{\prime \prime}(s)-\left(\int_{s^{\prime}=s}^{s^{\prime}=L} f\left(s^{\prime}\right) \cos \theta\left(s^{\prime}\right) \mathrm{d} s^{\prime}\right) \cos \theta(s) \\
-\left(\int_{s^{\prime}=s}^{s^{\prime}=L} f\left(s^{\prime}\right) \sin \theta\left(s^{\prime}\right) \mathrm{d} s^{\prime}\right) \sin \theta(s)=0 .
\end{array}
$$

Note that this equation only depends on the angular variable $\theta(s)$. Eq. 3 must be solved together with the boundary condition: $\theta(s=0)=\pi / 2$. At $s=L$ the fiber end is free to move, $m(L)=0$. We have completely neglected the weight of the fiber. We also note that our filament description allows for very large deflection (geometrically exact). 


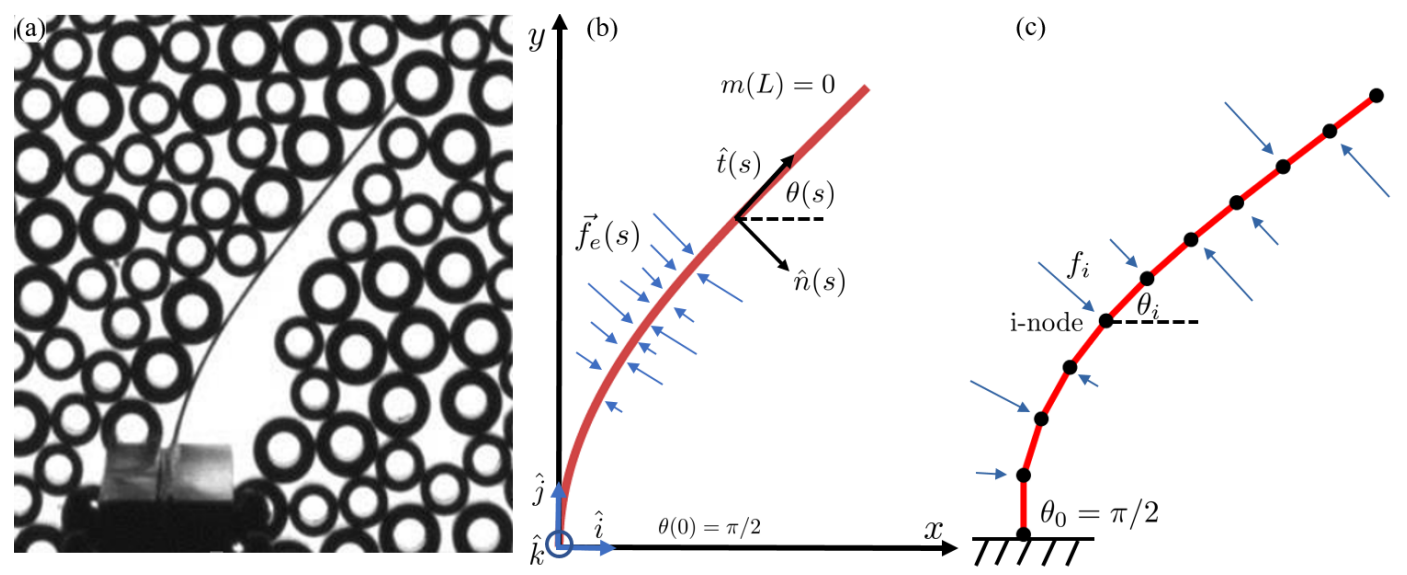

Figure 1. (a) Experimental example of a flexible fiber penetration into a granular medium (from [5]). Grains exert random forces at different fiber points. (b) Continuous fiber parametrization. Blue arrows represent the local force distribution $\vec{f}_{e}(s)$ for a normal loading with fluctuations of amplitude. (c) Discretized fiber parametrization. Random local forces (blue arrows) act in fiber nodes, where $\theta_{i}$ represents the angle of a given segment with respect to the horizontal axis.

The only restriction is that fiber self-intersections are forbidden.

\subsection{Numerical resolution}

Using finite differences, the second-order partial differential Eq. 3 can be discretized in a set of $\mathrm{N}$ nonlinear algebraic equations with $\mathrm{N}$ unknowns, that are the rotational degrees of freedom of the discretized structure, represented here by the angle $\theta_{i}$. Note that $\mathrm{N}$ is also the number of discrete elements modeling the fiber (see Fig.1(c)). In this way, the discrete, non-dimensional version of Eq. 3 is:

$$
\begin{array}{r}
-\frac{\left(\theta_{i+1}-2 \theta_{i}+\theta_{i-1}\right)}{d s^{2}}+\left(\sum_{j=i}^{j=N} f_{j} \cos \theta_{j}\right) \cos \theta_{i} \\
+\left(\sum_{j=i}^{j=N} f_{j} \sin \theta_{j}\right) \sin \theta_{i}=0 .
\end{array}
$$

In this last equation we keep the same notation for simplicity: $f_{i} \longrightarrow f_{i} / F_{C}$ and $s \longrightarrow s / L$, where $F_{C}=E I / L^{2}$ is the characteristic force of the problem. To solve this system of nonlinear equations we use MATLAB fsolve function, with the implemented trust-region-dogleg algorithm as an option. For all the performed simulations the number of segments has been set to $\mathrm{N}=200$ to ensure numerical convergence.

The force distribution along the fiber is the set of forces $\left\{f_{i}\right\}_{i=1}^{N}$. The classical problem considers that all the $f_{i}$ forces are uniformly distributed. We denote this configuration as deterministic solution. Thus, for a given force distribution and an initial configuration of the discrete beam $\left\{\theta_{o}^{i}\right\}_{i=1}^{N}$, we can obtain the quasistatic configuration $\left\{\theta^{i}\right\}_{i=1}^{N}$ which only depends on the applied force distribution.

Now, to account for the influence of force fluctuations, we suppose that all the $f_{i}$ are independent, identically distributed random variables. For simplicity, we consider that they are drawn from a Gaussian probability distribution:

$$
\mathcal{G}\left(f \mid \mu_{f}, \sigma_{f}\right)=\frac{1}{\sqrt{2 \pi \sigma_{f}^{2}}} \exp \left(\frac{\left(f-\mu_{f}\right)^{2}}{2 \sigma_{f}^{2}}\right),
$$

where $\mu_{f}$ is a prescribed mean value and $\sigma_{f}$ its standard deviation. For different fixed values of the couple $\left(\mu_{f}, \sigma_{f}\right)$ we have performed $N_{r}=200$ realizations of the random variables $\left\{f_{i}\right\}_{i=1}^{N}$. For each realization the shape of the fiber is constructed from the computed values of $\left\{\theta^{i}\right\}_{i=1}^{N}$.

To analyze the fiber deformations we can first focus on two quantities. The non-dimensional contact force acting as a reaction of the external forces at the clamped origin: $f_{T}=\sum_{i=1}^{N} f_{i}$. The second quantity is the reaction moment at the clamped end produced by these forces. By definition it can be computed by: $m_{o}=m(0)=E I K(0)$. In the special case of small deflection regime, the normal forces have essentially only horizontal components and then they are initially acting at a distance $\left\{y_{i}\right\}_{i=1}^{N}$ from the fiber origin. Therefore the non-dimensional total moment at the clamped end can be easily computed as: $m_{L o}=-\sum_{i=1}^{N} y_{i} f_{i}$ and this equation can be used to analyze the role of spatial fluctuations in the case of small random force amplitudes acting on the fiber.

\section{Results}

\subsection{Low and moderate fluctuations}

We discretized the fiber in $\mathrm{N}=200$ segments. At a given value of the couple $\left(\mu_{f}, \sigma_{f}\right)$ we performed $N_{r}=200$ realizations for all the simulations. To graphically see the effects of force amplitude fluctuations, in Fig. 2(a,b) we show some examples of fiber configurations obtained by solving numerically Eq. 4 using a distribution of forces given by Eq. 5. For this Fig. 2(a,b), we have fixed 2 values of $\mu_{f}=0.0$ and 0.1 . Each different figure corresponds to a different value of $\sigma_{f}=0.1$ and 1 ((a) and (b), respectively). We observe that for small fluctuations $\left(\sigma_{f}=0.1\right)$ the shapes coincide with the deterministic configurations obtained by the corresponding homogeneous distribution 

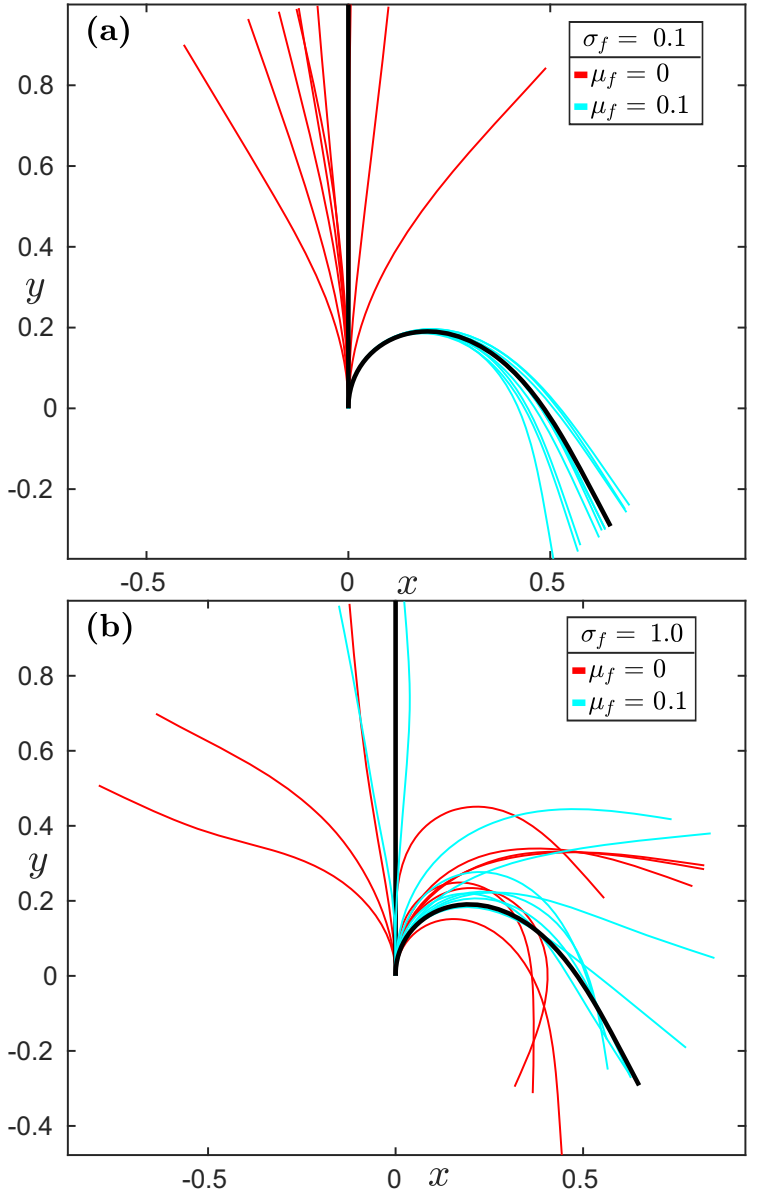

Figure 2. The influence of the noise magnitude on fiber configurations for 10 realizations (of a total of 200) for two fixed values of $\mu_{f}$. We show results for 2 different values of $\sigma_{f}$ : a) $\sigma_{f}=0.1$, b) $\sigma_{f}=1.0$. In both figures the black curve corresponds to the configuration obtained for the homogeneous distribution of forces, i.e., $\sigma_{f}=0$.

of forces (black curves, $\sigma_{f}=0$ ), at least when the curvature of the beam is large enough $\left(\mu_{f}=0.1\right)$. We also observe that when fluctuations start to increase $\left(\sigma_{f}=1\right)$ shapes are no longer similar and slightly disordered configurations appear for any shape (see Fig. 2(b)).

In Fig. 3 we show the behavior of the non-dimensional value of the moment at the origin $m_{o}$ as a function of the non-dimensional total force $f_{T}$. We observe the evolution of the curve for a given set of $\mu_{f}$ values (see inset in Fig. 3(a) and (b)) and for the same two fixed values of $\sigma_{f}=0.1$ and 1.0. For small fluctuations values (Fig. 3(a)) random results follow the deterministic curve represented by open black circles. Black crosses correspond to the mean value of all the realizations, each of one depicted by a color point of the scattered curve. We also include the corresponding values of the moment obtained in the small deformation regime by using the definition of $m_{L o}$ (blue squares) and its corresponding mean value (blue stars). We can see that the linear regimes are similar up to $\mu \approx 0.05$. Beyond this value non linear deformations dominate. Note that crosses and circles (and correspondingly squares and stars) almost coincide. Conversely, for moderately large
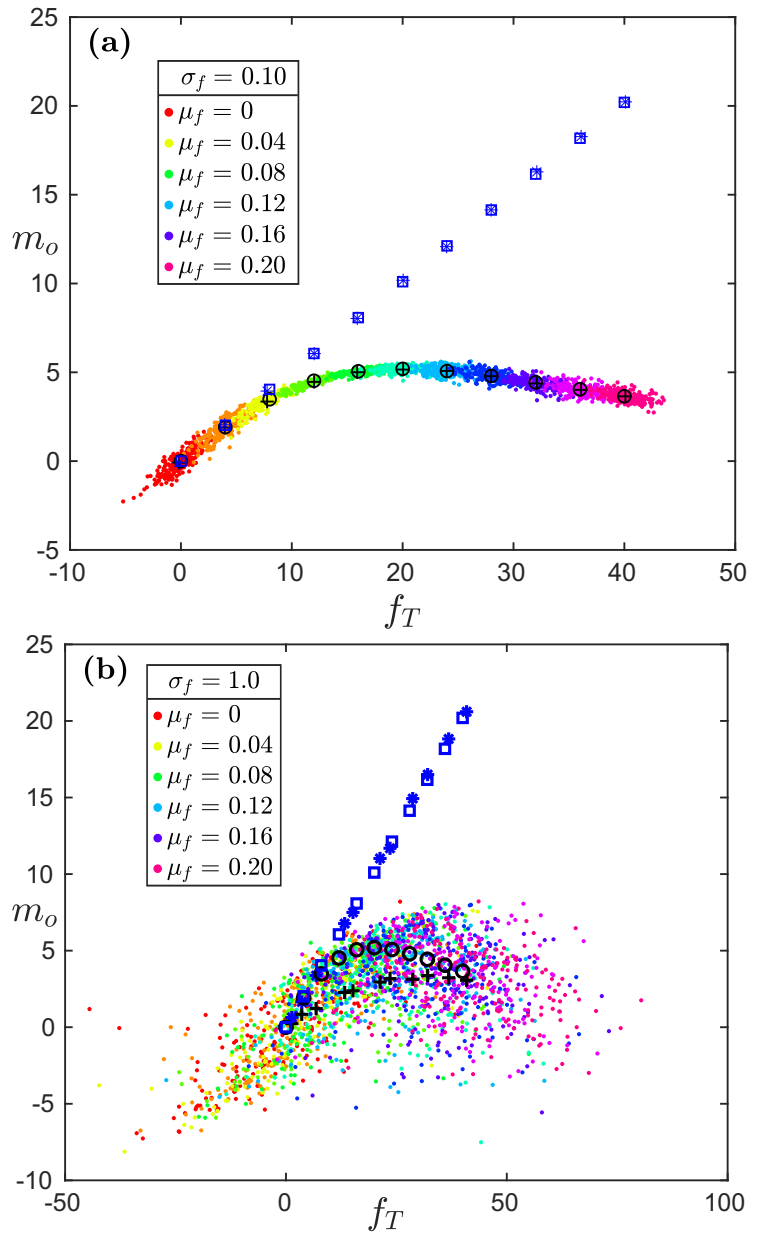

Figure 3. The influence of noise magnitude on the value of $m_{o}$, the fiber bending moment at $s=0$ for some fixed values of $\mu_{f}$ (see inset of each figure). We show results for 2 different noise values. a) $\sigma_{f}=0.1$, b) $\sigma_{f}=1.0$. Open black circles represent deterministic configurations and black crosses correspond to the mean value of all realizations. The value of $m_{L o}$ (small deformation limit) has been also plotted for the corresponding deterministic (blue squares) and realization mean values (blue stars).

fluctuations (Fig. 3(b)) shapes are completely uncorrelated to their corresponding deterministic configuration: mean and deterministic values are very far from each other and also with respect to the scattered points (realizations). This is associated with random fiber shapes that do not even follow the deterministic configuration trend, even for large curvature value (Fig. 2b).

\subsection{Large Fluctuation}

In the previous results, we show that for small fluctuations, the curvature of random and deterministic configurations follows the same trend (see Fig. 3(a)), in particular when the corresponding deterministic (initial) configurations have a large curvature $\left(\mu_{f}>0\right)$. We also observe that for moderately large fluctuations $\left(\sigma_{f} \approx 1.0\right)$, the geometrical parameters such as $m_{o}$ diverges from the ones of the deterministic configuration. This can be also observed for the mean values of realizations. 

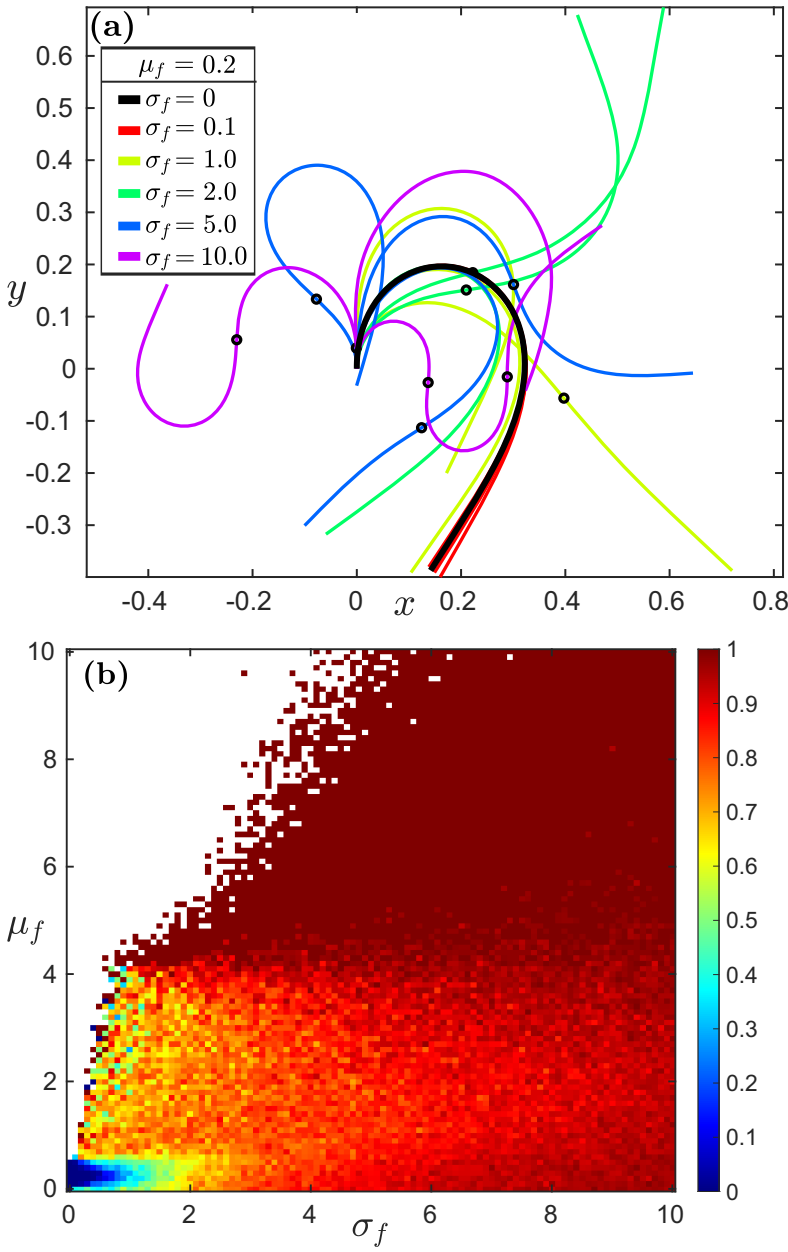

Figure 4. (a) Some examples of disordered realizations for different values of $\sigma_{f}$ at a given value of $\mu_{f}=0.2$. Open black circles represent the points on the fiber where curvature sign changes. (b) Relative proportion of fiber realizations whose curvature has changed at least once, as a function of $\left(\mu_{f}, \sigma_{f}\right)$. White regions correspond to self-intersecting fibers.

To quantify when fluctuations are large enough to influence the fiber configurations we can examine the behavior of their curvature. In Fig. 4(a) we observe the configurations obtained at fixed value of $\mu_{f}=0.2$ for increasing values of the noise $\sigma_{f}$. We have plotted 3 realizations for each corresponding value of parameters. Very complex shapes are observed, completely uncorrelated with the corresponding deterministic value (continuous black curve) open black circles correspond to points on the configuration were the fiber curvature has changed its sign. For certain situations and depending on the used parameters we can have, 1, 2, 3 or even more curvature sign changes in fibers where originally the deterministic shape has not. For all the performed realizations at fixed couple of $\left(\mu_{f}\right.$, $\sigma_{f}$ ) values, self-intersecting curves have been discarded from our analysis.
To get more insight into this behavior we performed a phase diagram describing the possible curvature sign changes for a region $\left(\mu_{f}, \sigma_{f}\right)$. In Fig. 4(b) we show a color map representing the relative proportion of realizations for which there is at least one curvature sign change $\left(\mathcal{P}_{\kappa C}\right)$. This proportion is relative because it has been computed by only considering fibers with a given number of curvature sign change with respect to all the physically accepted configurations. Note that this number is not necessarily equal to the corresponding realizations number $\left(N_{r}=200\right)$.

We observe a big region for which all the fibers are self-contacting, which is in fact obvious at large values of $\mu_{f}$ and moderately large values of $\sigma_{f}$ (see white region in Fig. 4(b)). Conversely, the region for which $0 \leq \mu_{f} \leq 0.5$ and $0 \leq \sigma_{f} \leq 1.0$, the proportion $\mathcal{P}_{\kappa C}$ is low, which means that no curvature sign changes are produced in this region. This is consistent with the results observed in Fig. 2(a) and Fig. 3(a).

\section{Conclusions}

We study the influence of small, moderate and large fluctuations on the deformations of a fiber subjected to local randomly distributed forces. We have analyzed different quantities representing the fiber mechanics, such as its total force and bending moment at the clamping point. The disorder in shape configurations seems to depend on the curvature of the corresponding deterministic shape and the amplitude in force fluctuations. For very large fluctuations, random and deterministic shapes are completely uncorrelated, but curvature sign changes seem to follow a particular behavior. This modeling of the noise in the loading forces on the fiber is a first step towards the understanding of the shapes adopted by a slender elastic object interacting mechanically with a granular medium.

\section{References}

[1] A. Huynen, E. Detournay, V. Denoel, P R Soc A 472 (2016)

[2] E. Kolb, V. Legué, M.B. Bogeat-Triboulot, Phys Biol 14, 065004 (2017)

[3] C.P. Brangwynne, F.C. MacKintosh, S. Kumar, N.A. Geisse, J. Talbot, L. Mahadevan, K.K. Parker, D.E. Ingber, D.A. Weitz, J Cell Biol 173, 733 (2006)

[4] M.R. Shaebani, J. Najafi, A. Farnudi, D. Bonn, M. Habibi, Nat Commun 8, 1 (2017)

[5] N. Algarra, P. Karagiannopoulos, A. Lazarus, D. Vandembroucq, E. Kolb, Phys Rev E 97, 022901 (2018)

[6] S. Antman, Nonlinear Problems of Elasticity (Springer New York, 1995)

[7] B. Audoly, Y. Pomeau, Elasticity and Geometry: From Hair Curls to the Non-linear Response of Shells (Oxford, 2010) 